\title{
Auditory-motor adaptation is reduced in adults who stutter but not in children who stutter
}

\author{
Ayoub Daliri $^{1,2}$ | Elizabeth A. Wieland ${ }^{3}$ | Shanqing Cai ${ }^{4}$ | Frank H. Guenther ${ }^{1}$ | \\ Soo-Eun Chang ${ }^{5}$
}

${ }^{1}$ Department of Speech, Language, and Hearing Sciences, Boston University, Boston, MA, USA

${ }^{2}$ Department of Speech and Hearing Science, Arizona State University, Tempe, AZ, USA

${ }^{3}$ Department of Communicative Sciences \& Disorders, Michigan State University, East Lansing, MI, USA

${ }^{4}$ Google, Cambridge, MA, USA

${ }^{5}$ Department of Psychiatry, University of Michigan, Ann Arbor, MI, USA

\section{Correspondence}

Ayoub Daliri, Department of Speech, Language, and Hearing Sciences, Sargent College of Health and Rehabilitation Sciences, Boston University, 677 Beacon Street, Room 102, Boston, MA 02215, USA.

Email: Ayoub.daliri@gmail.com

Funding information

National Institute on Deafness and Other Communication Disorders, Grant/Award Number: R01DC011277 and R01DC007683

\begin{abstract}
Previous studies have shown that adults who stutter produce smaller corrective motor responses to compensate for unexpected auditory perturbations in comparison to adults who do not stutter, suggesting that stuttering may be associated with deficits in integration of auditory feedback for online speech monitoring. In this study, we examined whether stuttering is also associated with deficiencies in integrating and using discrepancies between expected and received auditory feedback to adaptively update motor programs for accurate speech production. Using a sensorimotor adaptation paradigm, we measured adaptive speech responses to auditory formant frequency perturbations in adults and children who stutter and their matched nonstuttering controls. We found that the magnitude of the speech adaptive response for children who stutter did not differ from that of fluent children. However, the adaptation magnitude of adults who stutter in response to auditory perturbation was significantly smaller than the adaptation magnitude of adults who do not stutter. Together these results indicate that stuttering is associated with deficits in integrating discrepancies between predicted and received auditory feedback to calibrate the speech production system in adults but not children. This auditory-motor integration deficit thus appears to be a compensatory effect that develops over years of stuttering.
\end{abstract}

\section{RESEARCH HIGHLIGHTS}

- We examined sensorimotor adaptation in children and adults who stutter.

- The magnitude of the speech adaptive response for children who stutter did not differ from that of fluent children.

- The magnitude of adaptation of adults who stutter was significantly smaller than that of adults who do not stutter.

- Auditory-motor adaptation is reduced in adults who stutter but not in children who stutter.

\section{1 | INTRODUCTION}

Developmental stuttering is a disorder that disrupts the fluency of speech. The typical onset of stuttering is between 2 and 3 years of age (Yairi \& Ambrose, 2013). Stuttering affects approximately 4-5\% of preschool children, and the disorder persists into adulthood in approximately $20-30 \%$ of these children (e.g., Yairi \& Ambrose, 1999). The prevalence of stuttering is close to $1 \%$ in the general population (e.g., Yairi \& Ambrose, 2013). Over the past decades, many studies have examined neural mechanisms of stuttering in adults who stutter and have proposed that stuttering may be associated with deficits in the sensorimotor system (Bloodstein \& Bernstein-Ratner, 2008). However, an important question that has received little attention is whether deficits in the sensorimotor system are present in children who stutter (i.e., whether the deficits are primary factors in the emergence of stuttering during childhood).

Many neuroimaging studies investigating underlying neural deficits of stuttering have reported abnormal brain activation in auditory and motor regions of adults who stutter (Chang, Horwitz, Ostuni, Reynolds, \& Ludlow, 2011; Fox et al., 1996; Kell et al., 2009; Chang, 
Kenney, Loucks, \& Ludlow, 2009; Watkins, Smith, Davis, \& Howell, 2008). In addition, structural neuroimaging studies have revealed abnormal connectivity between speech motor and auditory regions (Cai, Beal, Ghosh, Guenther, \& Perkell, 2014; Chang et al., 2011; Cykowski, Fox, Ingham, Ingham, \& Robin, 2010; Sommer, Koch, Paulus, Weiller, \& Buchel, 2002; Watkins et al., 2008). The extent of these effects vary across studies and individuals (Cykowski et al., 2010; Wymbs, Ingham, Ingham, Paolini, \& Grafton, 2013); however, the preponderance of evidence has led to a suggestion that abnormality in auditory and speech motor neural networks may result in deficits in integration of auditory feedback for speech production (Beal et al., 2010; Cai et al., 2014; Chang et al., 2011; Daliri \& Max, 2015a, 2015b; Max, 2004; Max, Guenther, Gracco, Ghosh, \& Wallace, 2004).

Consistent with this view, many behavioral studies have investigated auditory-motor integration of adults who stutter during speech production (Bloodstein \& Bernstein-Ratner, 2008). It is well known that artificial changes in auditory feedback, such as delayed auditory feedback, can induce fluency in people who stutter (Foundas, Mock, Corey, Golob, \& Conture, 2013; Kalinowski, Stuart, Sark, \& Armson, 1996; Lee, 1951; Wingate, 1969; Yates, 1963). Furthermore, people with more severe stuttering benefit more from these 'fluency enhanc ing' conditions compared to those with less severe stuttering (Foundas et al., 2013; Lincoln, Packman, \& Onslow, 2006). These studies suggested that stuttering may be associated with deficits in auditorymotor integration. However, the coarse nature of these manipulations of the auditory feedback may not have served as adequate probes for detailed investigation of the role of the auditory feedback in speech production of people who stutter. Thus, subsequent behavioral studies have examined this deficit in more detail using perturbation techniques to systematically manipulate specific parameters of the auditory feedback (Bauer, Seery, LaBonte, \& Ruhnke, 2007; Cai et al., 2014; Cai, Beal, Ghosh, Tiede, Guenther, \& Perkell, 2012; Loucks, Chon, \& Han, 2012; Nudelman, Herbrich, Hess, Hoyt, \& Rosenfield, 1992). Both prior neuroimaging and behavioral studies, however, have generally examined neural mechanisms of stuttering in adults who stutter, leaving open the possibility that deficits in auditory-motor integration are secondary compensatory behaviors developed over years of stuttering, rather than core deficits associated with stuttering.

Auditory perturbation has been employed in two different experimental designs, each investigating distinct aspects of auditory-motor integration: unexpected perturbation and sustained perturbation. In unexpected perturbation, the perturbation is applied on a random trial or at a random time during a trial (Burnett, Senner, \& Larson, 1997; Cai, Ghosh, Guenther, \& Perkell, 2011; Elman, 1981; Larson, Burnett, Bauer, Kiran, \& Hain, 2001; Purcell \& Munhall, 2006a; Tourville, Reilly, $\&$ Guenther, 2008). Typically, speakers produce a reflexive compensatory response to the perturbation (usually in the opposite direction of the perturbation and with 100-200 ms lag relative to the onset of the perturbation). According to current theories of speech production (Guenther, 2006; Hickok, 2012; Hickok, Houde, \& Rong, 2011; Tourville \& Guenther, 2011), the sensorimotor system uses motor-toauditory mappings to predict auditory consequences of issued motor commands that generate articulatory movements. Then, during speech production, the sensorimotor system compares predicted auditory consequences of the issued motor commands with the received (i.e., actual) auditory feedback. When a discrepancy between the predicted and received feedback exists - as is the case during unexpected auditory perturbations - the sensorimotor system generates a corrective motor response to compensate for the effects of perturbations. Thus, using such a paradigm, one can investigate the integration of auditory feedback for online monitoring and control of the speech production system (Cai et al., 2011; Tourville et al., 2008).

In the second type of paradigm, the perturbation is applied on a set of successive trials; participants gradually develop/learn an adaptive response (i.e., long-term change in their production) to compensate for the perturbation (Houde \& Jordan, 1998; Houde \& Jordan, 2002; Jones \& Munhall, 2000, 2002; Katseff, Houde, \& Johnson, 2012; MacDonald, Goldberg, \& Munhall, 2010; Max \& Maffett, 2015; Purcell \& Munhall, 2006b; Rochet-Capellan, Richer, \& Ostry, 2012; Vaughn \& Nasir, 2015; Villacorta, Perkell, \& Guenther, 2007). The adaptation effect does not disappear immediately after the termination of the perturbation when participants revert to hearing unperturbed auditory feedback; this indicates a learned (rather than reflexive) response in which stored motor programs have been updated to incorporate compensatory adjustments. Using an adaptation paradigm, one can investigate the integration of auditory feedback for calibration and maintenance of the speech production system (Houde \& Jordan, 1998; MacDonald et al., 2010; Purcell \& Munhall, 2006b). The extent of the adaptive response to auditory perturbations can vary widely across individuals, and for most individuals the adaptation is not complete (i.e., the magnitude of the adaptive response is less than the extent of perturbations). It has been suggested that the individual variability and incompleteness of the adaptive response may be explained by the extent of reliance on auditory feedback (that is perturbed) and somatosensory feedback - individuals who rely more on auditory feedback may adapt more than those who rely more on somatosensory feedback (Feng, Gracco, \& Max, 2011; Katseff et al., 2012; Lametti, Nasir, \& Ostry, 2012; Perkell, 2012).

In a previous study, Cai and colleagues (2012) examined the magnitude of compensatory response to unexpected formant perturbation in adults who do and do not stutter. The results showed that compensatory responses of adults who stutter were significantly reduced compared to controls. Similarly, this limited compensatory response in adults who stutter has been reported in response to unexpected perturbation of other speech parameters such as fundamental frequency (Bauer et al., 2007; Loucks et al., 2012; Nudelman et al., 1992) and temporal parameters of articulation (Cai et al., 2014). Together, these studies suggest that adults who stutter are inefficient in integrating auditory feedback for online monitoring and producing appropriate corrective motor responses to compensate for auditory perturbation. It has been suggested that the inefficiency in the auditory-motor integration (or sensory-motor integration in general) of adults who stutter may be a result of reduced reliance on auditory feedback (or sensory feedback in general) (see Daliri, Prokopenko, \& Max, 2013; Loucks $\&$ De Nil, 2006b; Max, 2004). However, it remains unclear whether the possible reduced reliance on auditory feedback may also result in 
difficulties in using auditory feedback for calibration and maintenance of speech production in adults who stutter. In other words, adults who stutter may have difficulties in using discrepancies - experimentally generated by the auditory perturbation - between the predicted auditory consequences of the planned articulatory movements and received auditory feedback to produce appropriate adaptive responses to the perturbation. This is an especially intriguing question, as several recent studies have reported that both adults and children who stutter are less efficient in integrating discrepancies between sensory goals and sensory feedback during the production of new motor skills (i.e., improving their performance with practice) and also less efficient in retaining the newly-learned motor skills (Bauerly \& De Nil, 2011; Olander, Smith, \& Zelaznik, 2010; Sasisekaran, Smith, Sadagopan, \& Weber-Fox, 2010; Smits-Bandstra, De Nil, \& Rochon, 2006; SmitsBandstra, De Nil, \& Saint-Cyr, 2006; Smits-Bandstra \& De Nil, 2009). Together these studies suggest that stuttering may be associated with deficiencies in using and integrating discrepancies between predicted and received feedback to adaptively update the speech motor system.

In the present study, an adaptation paradigm was used to experimentally generate a discrepancy between predicted auditory feedback and received auditory feedback in order to examine whether stuttering is associated with deficits in integration of auditory feedback (i.e., using discrepancies in feedback) to adaptively update motor programs for speech. In addition, an important question is whether the possible deficits in auditory-motor integration shown in adults who stutter are also evident in children who stutter. For the first time, this crucial developmental question is examined by investigating auditory-motor integration in children who stutter. Thus, it is hypothesized that (a) if stuttering is associated with deficits in auditory-motor integration, adults who stutter would have a smaller adaptive response to sustained auditory perturbation compared to adults who do not stutter, and (b) if a deficit in auditory-motor integration is a contributing factor in the emergence of stuttering during childhood, children who stutter would have a smaller adaptive response to auditory perturbation in comparison to children who do not stutter.

\section{2 | METHODS}

\section{1 | Participants}

Four groups of participants were recruited: 20 children who do not stutter (CNS; 11 males; age: $M=8.63$ years, $S D=1.42$; range $=7.08$ 11.42), 20 children who stutter (CWS; 11 males; age: $M=8.49$ years, $S D=1.51 ;$ range $=6.08-11.17$ ), 14 adults who do not stutter (ANS; 8 males; age: $M=23.74$ years, $S D=6.92$; range $=18.75-43.75$ ), and 14 adults who stutter (AWS; 12 males; age: $M=30.63$ years, $S D=12.44$; range $=18.08-53.08$ )

Eligibility criteria for all participants included: (a) being a monolingual, native speaker of American English, (b) self-reported (for adults) or parent-reported (for children) absence of developmental, psychological, neurological, or communication disorders (other than stuttering in the participants who stutter), (c) normal binaural hearing thresholds ( $\leq 20 \mathrm{~dB} \mathrm{HL}$ for children, $\leq 25 \mathrm{~dB}$ HL for adults) at 1000,2000 , and
$4000 \mathrm{~Hz}$, (d) not taking any medications that affect the central nervous system, and (e) scoring at the 20th percentile or higher on standardized speech, language, and cognitive tests (described below). All participants were naïve to the purpose of the study, and they signed informed consent prior to participation (all children signed consent along with their parents). The study was approved by the Michigan State University Institutional Review Board.

To ensure that all participants demonstrated age-appropriate speech and language abilities, we used a battery of standardized speech, language, and cognitive tests prior to participation in the study. The battery included (a) the Peabody Picture Vocabulary test (PPVT-4; Dunn \& Dunn, 2007), (b) the Goldman Fristoe Test of Articulation (GFTA-2; Goldman \& Fristoe, 2000), (c) the Expressive Vocabulary Test (EVT-2; Williams, 2007), (d) receptive language tests for children only (subtests within the Test of Language Development, TOLD-P:3, ages 4;0-8;11; TOLD-14, ages 9;0-12;0; or Test for Auditory Comprehension of Language, TACL-3, ages 4;0-8;11 (Carrow-Woolfolk, 1999; Hammill, Brown, Larsen, \& Wiederholt, 1994; Hammill \& Newcomer, 1988; Newcomer \& Hammill, 1977)), and (e) the Wechsler Abbreviated Scale of Intelligence for children only (WASI; Wechsler, 1999). We additionally used the Edinburgh handedness inventory (Oldfield, 1971) to determine the handedness of participants.

Video-recorded samples of speech tasks - conversational speech (conversations with a clinician), monologue (storytelling narrative elicited via a wordless picture book for children; Mayer, 1969), and reading task - were reviewed off-line by an American SpeechLanguage-Hearing Association-certified speech-language pathologist (SLP) to confirm diagnosis and severity of stuttering. The Stuttering Severity Instrument, Fourth Edition (SSI-4; Riley, 2008) was used to determine stuttering severity for both AWS and CWS, based on a minimum 500-syllable speech sample recorded during the speech tasks described above. In order for a child to be considered in the stuttering group, the child needed to (a) exhibit at least 3\% stuttering-like disfluencies, (b) score at least 'very mild' based on the composite SSI-4 score, and (c) be considered stuttering based on both parent report and clinician impressions. Based on two independent SLPs' SSI ratings, we calculated an intra-class correlation as a measure of coefficient reliability of the SSI score ratings. There was a high agreement between the two independent SLPs (Cronbach's alpha $=0.97$ ). Information regarding age, sex, total SSI score, and SSI stuttering severity classification for CWS and AWS are listed in Tables 1 and 2, respectively.

\section{2 | Apparatus}

The experiment was conducted in a quiet room with participants seated in front of a 23-in computer monitor. The monitor was used to present pictures that elicited the target words 'head', 'Ted', or 'bed' (Figure 1A), and also to provide visual feedback about the participant's speech intensity and duration after completion of each trial.

Figure $1 \mathrm{~A}$ depicts the apparatus of the experiment. During the experiment, participants were wearing headphones (HD 380 PRO, Sennheiser Electronic Corporation, Old Lyme, CT) with a headmounted microphone (AT-803, Audio-Technica, Stow, OH) that was 
TABLE 1 Information regarding age, sex, total SSI score, and SSI stuttering severity classification for children who stutter (CWS)

\begin{tabular}{|lllll|}
\hline ID & Age (years) & Sex & $\begin{array}{l}\text { Total SSI } \\
\text { score }\end{array}$ & Severity \\
\hline CWS1 & 9.92 & female & 29 & severe \\
\hline CWS2 & 10.00 & female & 19 & mild \\
\hline CWS3 & 8.00 & female & 16 & mild \\
\hline CWS4 & 10.83 & female & 8 & very mild \\
\hline CWS5 & 8.75 & female & 13 & mild \\
\hline CWS6 & 6.08 & female & 22 & moderate \\
\hline CWS7 & 9.83 & female & 17 & mild \\
\hline CWS8 & 7.33 & female & 16 & mild \\
\hline CWS9 & 9.67 & female & 24 & moderate \\
\hline CWS10 & 11.17 & male & 14 & mild \\
\hline CWS11 & 7.25 & male & 10 & very mild \\
\hline CWS12 & 10.25 & male & 11 & mild \\
\hline CWS13 & 7.00 & male & 23 & moderate \\
\hline CWS14 & 7.58 & male & 21 & moderate \\
\hline CWS15 & 7.08 & male & 13 & mild \\
\hline CWS16 & 7.25 & male & 13 & mild \\
\hline CWS17 & 9.50 & male & 12 & mild \\
\hline CWS18 & 7.17 & male & 19 & mild \\
\hline CWS19 & 7.25 & male & 27 & moderate \\
\hline CWS20 & 7.83 & male & 25 & moderate \\
\hline
\end{tabular}

TABLE 2 Information regarding age, sex, total SSI score, and SSI stuttering severity classification for adults who stutter (AWS)

\begin{tabular}{|lllll|}
\hline ID & Age (years) & Sex & $\begin{array}{l}\text { Total SSI } \\
\text { score }\end{array}$ & Severity \\
\hline AWS1 & 21.83 & female & 20 & mild \\
\hline AWS2 & 45.25 & female & 16 & very mild \\
\hline AWS3 & 21.50 & male & 35 & moderate \\
\hline AWS4 & 44.08 & male & 18 & mild \\
\hline AWS5 & 19.33 & male & 24 & mild \\
\hline AWS6 & 18.08 & male & 26 & moderate \\
\hline AWS7 & 21.75 & male & 35 & severe \\
\hline AWS8 & 19.67 & male & 19 & mild \\
\hline AWS9 & 45.08 & male & 30 & moderate \\
\hline AWS10 & 53.08 & male & 37 & severe \\
\hline AWS11 & 22.83 & male & 14 & very mild \\
\hline AWS12 & 24.67 & male & 23 & mild \\
\hline AWS13 & 28.50 & male & 36 & severe \\
\hline AWS14 & 43.25 & male & 34 & severe \\
\hline
\end{tabular}

placed approximately $10 \mathrm{~cm}$ away from the corner of the participant's mouth. The signal from the microphone was amplified, digitized, and transferred to a Lenovo Thinkpad laptop (Intel ${ }^{\circledR}$ Core $^{\mathrm{TM}}$ i5 CPU) via an external audio interface (MOTU MicroBook, MOTU, Cambridge,
(A)

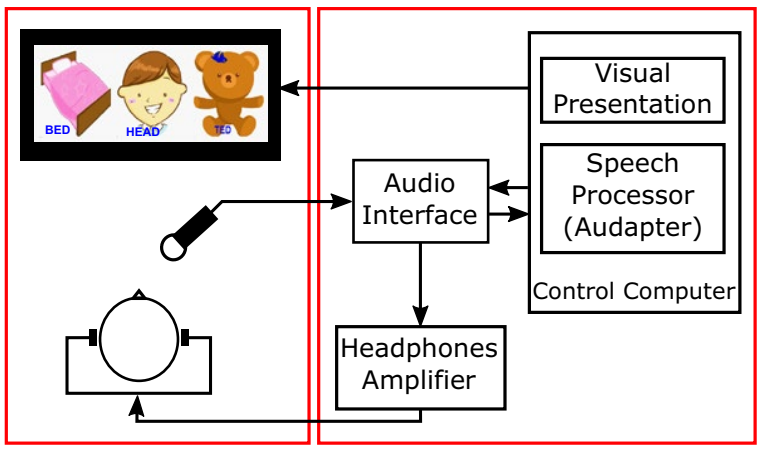

(B)

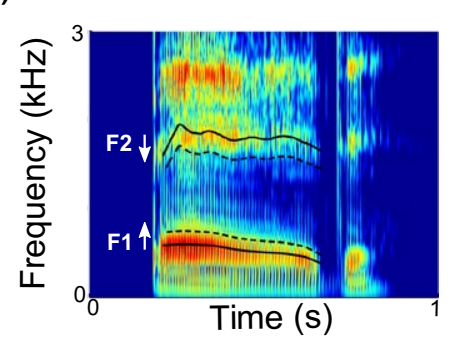

(C)

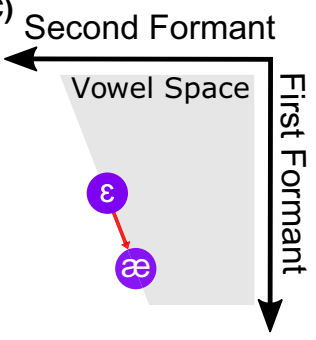

(D)

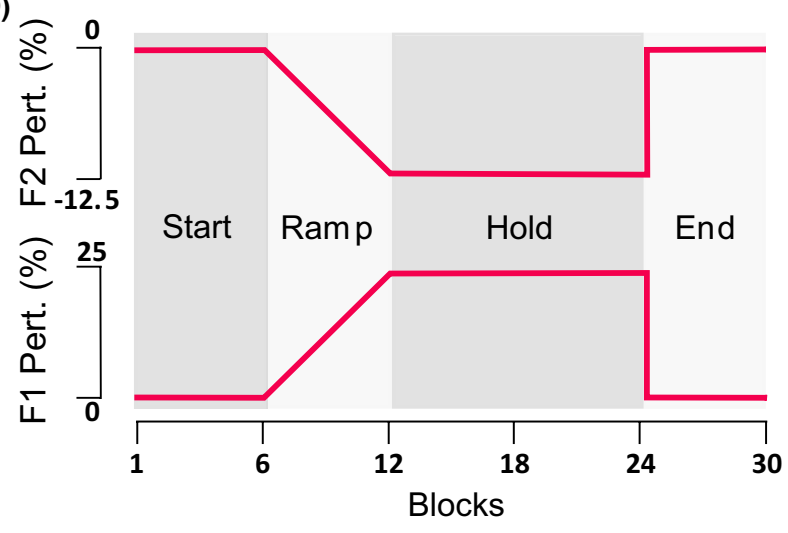

FIGURE 1 Apparatus (A) and procedure (D) of the experiment. During the experiment, after the Start phase (no perturbation), the magnitude of the perturbation was gradually modified - increase in the first formant (F1) and decrease in the second formant (F2) until it reached the maximum perturbation ( $25 \%$ increase in F1 and $12.5 \%$ decrease in F2). Then, during the Hold phase, the magnitude of perturbation was kept constant; during the End phase, no perturbation was applied. The spectrogram of the word 'bed' is shown in panel B. Solid lines correspond to unperturbed F1 and F2, and dashed lines correspond to the perturbed version of F1 and F2. The perturbations of vowel $/ \varepsilon$ / (presented as a red arrow in panel C) resulted in a signal with formant frequencies that were closer to formant frequencies of the vowel /æ/ in the vowel space.

MA). Audapter (Cai, 2015), a publicly available software, was used for speech processing and manipulation in near-real time ( 14 ms delay). Detailed descriptions of Audapter have been reported in previous publications (Cai, Ghosh, Guenther, \& Perkell, 2010; Cai et al., 2011). Briefly, the microphone signal was down-sampled to $12,000 \mathrm{~Hz}$ for real-time processing (to decrease computational loads and thus decrease the input-output delay). Formant frequencies were estimated every 2 ms using a combination of an autoregressive linear predictive coding algorithm and a dynamic-programming tracking algorithm, 
implemented in Microsoft Visual $\mathrm{C}++$. In a given trial, the estimated formant frequencies were then shifted based on the magnitude of perturbations in that trial (e.g., 25\% upward shift in the first formant). The shifted formant frequencies were used to synthesize the output signal (i.e., auditory feedback). The output signal of the Audapter was transferred via the audio interface, amplified (Xenyx502, Behringer, Braintree, MA), and binaurally played back to the participant through headphones.

\section{3 | Procedure}

The experiment was presented in the form of a game presented on a computer screen. A blue bird landed on a picture associated with one of the three target words containing the vowel $/ \varepsilon /$ (i.e., 'head', 'bed', or 'Ted') and participants were instructed to say the name of that picture (Figure 1A). The order of selected target words (pictures) was randomized within each block of three trials. First, participants completed five blocks of practice trials (15 trials). Upon completion of each practice trial, participants received visual feedback regarding the intensity and duration of their production. Specifically, visual feedback to participant was presented as a loudspeaker that varied in color (corresponding to whether or not participant's voice intensity was in the range of 72-88 dB SPL), and as a ruler that varied in length (corresponding to whether or not the duration of participant's speech was in the range of 300-700 ms). Immediately after the practice trials, participants completed 25 blocks of three trials for which visual feedback about the intensity and duration of speech were only provided if they were outside the expected range.

The experiment consisted of four phases: Start, Ramp, Hold, and End (Figure 1D). The Start phase consisted of six blocks during which participants heard their own production without auditory perturbation while producing the words. In the first five blocks of the Start phase, participants always received visual feedback regarding the length and intensity of their produced speech whereas for the rest of trials they only received feedback if they performed outside the acceptable range. The Ramp phase consisted of six blocks during which auditory perturbations were applied. The magnitude of the perturbations was gradually increased until it reached the maximum amount of perturbation at the end of this phase. The perturbation consisted of $25 \%$ increase in the first formant (F1) and $12.5 \%$ decrease in the second formant (F2) of the vowel $/ \varepsilon /$ (Figure 1B). The perturbations of vowel $/ \varepsilon /$ resulted in a signal with formant frequencies that were closer to formant frequencies of the vowel $/ æ /$ in the vowel space (Figure 1C). The Hold phase consisted of 12 blocks during which the perturbation was held at the maximum. The End phase consisted of six blocks and during this phase no auditory perturbation was applied (similar to the Start phase). Data from all trials - including all trials from the Start phase and trials where the participant's intensity or duration were outside the range - were entered into the analysis.

\section{4 | Data and statistical analysis}

Prior to data analysis, all trials were inspected manually by raters blinded to perturbation condition (to avoid any bias and to enhance the reliability of the analyses), in order to exclude trials that contained production errors or gross formant-tracking errors. A custom-written MATLAB script (The MathWorks, Inc., Natick, MA) was used to extract $F 1$ and F2 trajectories from data output of Audapter. Measurement reliability of Audapter has been established in previous studies (Cai et al., 2010; Cai et al., 2011). The formant trajectories were smoothed using locally weighted linear regression. Then the average formant frequency was calculated from a window placed over the center of the vowel $(40-60 \%$ into the length of the vowel). Utterances that contained production errors and/or outlier formant frequencies - outside the range of 2 standard deviations - were excluded from further analyses. As a result, approximately $7 \%$ of all experimental utterances were excluded from analyses (CNS: $M=7.951 \%, S D=3.733$; CWS: $M=8.163 \%, S D=3.426$; ANS: $M=6.569 \%, S D=2.872$; AWS: $M=6.204 \%, S D=3.026$ ). There were no significant differences between the groups who do and do not stutter in the number of excluded trials ( $p>$.135). The extracted formant frequencies for the three trials within each block (i.e., three target words) were then averaged. We did not find between-group differences for children and adults for either the first formants and the second formants of unperturbed trials in the Start phase $(p>.52$ in all cases). Table 3 presents the average formant values for all four groups. Formant values in all blocks were divided by the average of formants of the last nine trials in the Start phase, and the formants were expressed as percentages (hereafter labelled normalized-F1 for the first formant and normalized-F2 for the second formant). This procedure eliminated the general effect of individual differences in formant frequencies during the Start phase - especially differences between children and adults (see Table 3). In addition, presenting the data in the unit of percentage would allow us to directly compare the

TAB LE 3 Average (standard deviation inside the parentheses) formant frequencies of trials in the Start phase (the first 18 trials; unperturbed trials) for all four groups. (CNS: children who do not stutter, CWS: children who stutter, ANS: adults who do not stutter, AWS: adults who stutter.) These data indicate that during the Start phase (during which no perturbations were applied), there were no between-group differences for either children or adult groups in their first and second formant productions

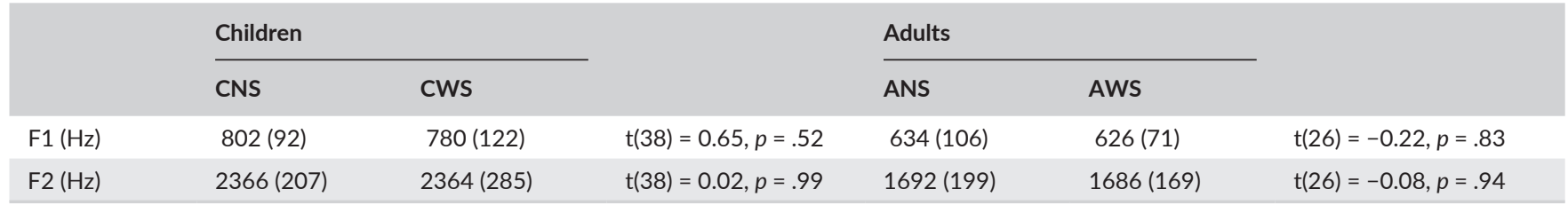


magnitude of perturbations, which is applied in the unit of percentage, and the magnitude of adaptive responses in each participant. Independent-samples $t$-tests (two-tailed) showed that vowel duration was not statistically significantly different between the groups who do and do not stutter ( $p=.842$ for adults, $p=.458$ for children). One should note that this lack of difference was expected as participants were trained to produce utterances with duration in the range of 300-700 ms (see Procedure).

Statistical analyses were conducted using IBM SPSS Statistics 20 (IBM, Armonk, NY). As dependent measures, the average normalized-F1 and normalized-F2 were calculated during the Start phase (blocks 4, 5, 6), the Hold phase (blocks 22, 23, 24), and the End phase (blocks 28, 29, 30). Separate repeated-measures analysis of variance (ANOVA) were conducted for the children and adults, with Group (stuttering, nonstuttering) as a between-subjects variable, and Phase (Start, Hold, End) as a within-subjects variable. Degrees of freedom were corrected using the Huynh-Feldt procedure to adjust for potential violations of sphericity assumption (Max \& Onghena, 1999). To examine homogeneity of variance across groups (CNS vs. CWS and ANS vs. AWS), we used Levene's test for equality of variances. Our results showed that the variance of our dependent variables did not differ significantly across groups ( $p>.105$ in all cases).

To ensure that participants actually changed their production in response to the perturbations, we carried out a priori planned comparisons (uncorrected paired sample $t$-tests) to examine whether significant differences existed between the Hold and Start phase for each group (Max \& Maffett, 2015; Quinn \& Keough, 2002; Ruxton \& Beauchamp, 2008). Statistically significant interactions were followed up with post-hoc analyses, using t-tests with Bonferroni corrections for multiple comparisons. In addition, we calculated Pearson correlation coefficients to examine possible relationships between dependent variables and participant characteristics (e.g., speech-language tests, stuttering severity).

\section{3 | RESULTS}

\subsection{Sensorimotor adaptation in adults}

Figure $2 \mathrm{~A}$ shows the group averaged normalized-F1 for adults. The results of $a$ priori tests that were conducted to examine adaptation

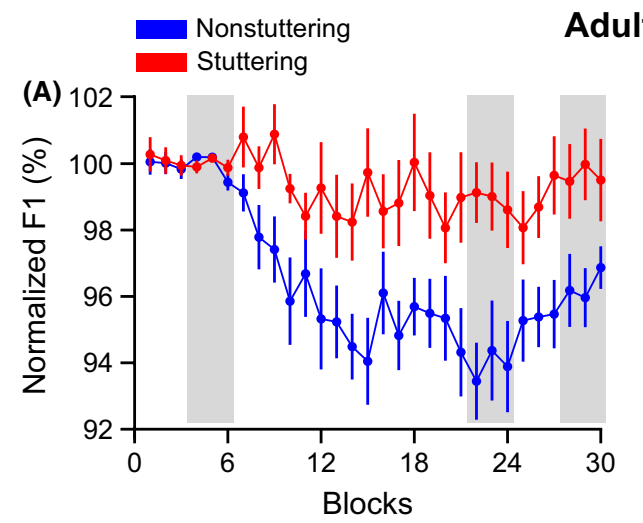

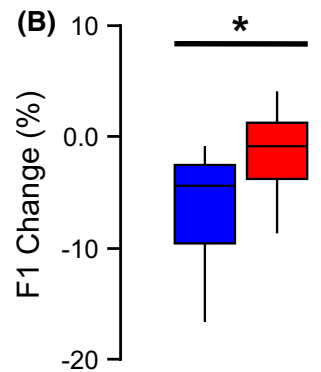

Hold Phase

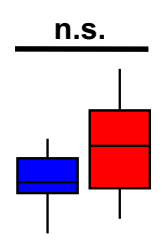

End Phase

Children

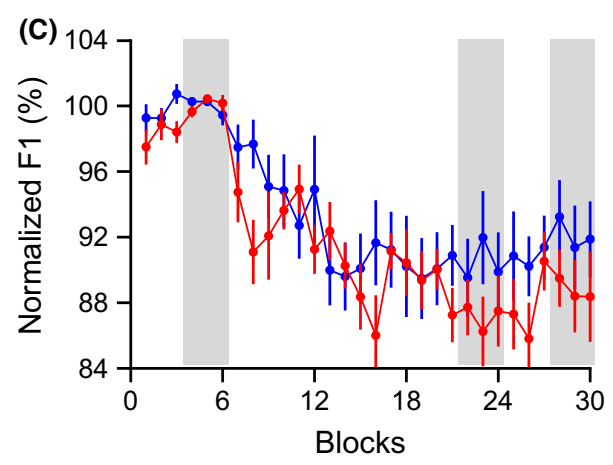

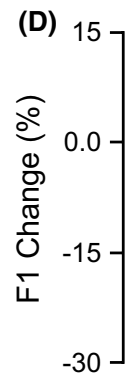

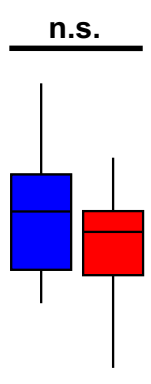

Hold Phase

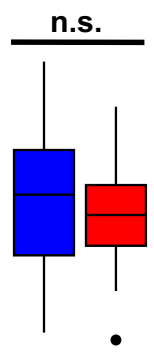

End Phase

FIGURE 2 The average normalized-F1 over the successive trials of the experiment are shown in panel A for adults and panel C for children (red for stuttering groups and blue for nonstuttering groups). The average data in the shaded areas were entered into the statistical analyses. Relative to their production in the Start phase, adults who do not stutter significantly changed their production (i.e., adapted) in response to the first formant perturbation in the Hold phase; however, this was not the case for adults who stutter (B). We also found that both children who do and do not stutter similarly adapted their production in the Hold phase relative to their production in the Start phase (see panels $C$ and D). Note that the scales of panels $A$ and $B$ (results for adults) are different from the scales of panels $C$ and $D$ (results for children). Comparing the extent of adaptation for adults who do not stutter with that for children who do not stutter, we found no statistically significant differences between the two groups $(p>$.134). However, comparing the groups who stutter revealed that children adapted more than adults ( $p<.001)$ in response to the perturbation of the first formant. Error-bars correspond to standard error of the mean. Asterisks represent $p<.01$ (two-tailed). ' $n s$ ' stands for statistically nonsignificant. 
(defined as the magnitude of change in normalized-F1 or normalized-F2 in the Hold phase relative to those in the Start phase; see Figure $2 \mathrm{~B}$ ) in the Hold phase showed that ANS successfully adapted to the perturbation in the first formant $(p<.001)$ but not to the perturbation in the second formant $(p=.426)$. However, AWS did not adapt to perturbations in the first formant $(p=.277)$ or the second formant $(p=.408)$. Furthermore, statistical analysis of the normalized-F1 data revealed statistically significant main effects of Condition, $F(2,52)=12.636, p<.001$, and Group, $F(1,26)=11.667$, $p=.002$, as well as a significant interaction of Condition by Group, $F(2,52)=6.232, p=.004$. This interaction indicated that (a) on average, the magnitude of adaptation for ANS was significantly greater than that for AWS $(p=.005)$, and (b) in contrast to ANS $(p<.001)$, however, AWS did not adapt to the perturbation in Hold phase in comparison to the Start phase ( $p=.381$ ) (see Figure $2 \mathrm{~B}$ ). It should be noted that both AWS and ANS performed similarly in the End phase $(p=.058)$. No statistically significant main effects of Condition, or Group, or Group by Condition interaction (in all cases $p>$.367) were found for normalized-F2.

Correlational analyses did not find any significant relationships between the dependent variables and any of the speech-language tests or stuttering severity measures.

\subsection{Sensorimotor adaptation in children}

The group averaged normalized-F1 for children is shown in Figure $2 \mathrm{C}$. The results of a priori tests showed that both CNS and CWS successfully adapted to the perturbation in the first formant $(p<.001)$ but not to the perturbation in the second formant $(p>.167)$. Furthermore, a statistically significant main effect of Condition was found for normalized- $F 1, F(1.547,58.791)=52.082, p<.001$, with similar pattern of response for the two groups (see Figure $2 \mathrm{C}$ and $\mathrm{D}$ ). However, our results did not reveal a significant main effect of Group or Group by Condition interaction (in both cases $p>.220$ ). Similar to the results for normalized-F1, we found a statistically significant main effect of Condition for normalized-F2, $F(1.433,54.462)=5.930, p=.010$, and no significant main effect of Group or Group by Condition interaction (in both cases $p>.126$ ).

Correlational analyses did not reveal significant relationships between the dependent variables and any of the speech-language tests or measures of stuttering severity.

\section{3 | Sensorimotor adaptation across groups}

Comparing the extent of adaptation (change in normalized-F1 or normalized-F2 in the Hold phase relative to those in the Start phase; see Figure $2 \mathrm{~B}$ and $\mathrm{D}$ ) for adults who do not stutter with that for children who do not stutter, we found no statistically significant differences between the extent of adaptation for both normalized-F1 and normalized-F2 ( $p>$.134). However, comparing the groups who stutter revealed that children adapted more than adults $(p<.001)$ in response to the perturbation of the first formant, but not to the perturbation of the second formant $(p=.237)$.

\section{4 | DISCUSSION}

Previous studies have shown that adults who stutter produce smaller corrective motor responses to compensate for unexpected auditory perturbations in comparison to adults who do not stutter, suggesting that stuttering may be associated with deficiencies in integrating auditory feedback for online feedback monitoring (Cai et al., 2014; Cai et al., 2012; Loucks et al., 2012; Nudelman et al., 1992). However, it remained unclear whether these deficiencies also result in difficulties using and integrating discrepancies between predicted auditory feedback and received auditory feedback to adaptively update motor programs for accurate speech production. Therefore, in the present study an adaptation paradigm was used to examine whether stuttering is associated with deficits in integrating discrepancies in auditory feedback for adaptive calibration of the speech motor system. Moreover, for the first time, we examined whether children who stutter also have similar auditory-motor integration deficits. We found that children who stutter did not differ from fluent children in adapting to the perturbation (25\% upward shift in F1 and 12.5\% downward shift in F2). However, we found that the magnitude of the adaptive response of adults who stutter in response to F1 perturbation was significantly smaller than the adaptive response of adults who do not stutter, whereas no difference was found between adaptive responses of the two groups for $\mathrm{F} 2$ perturbation.

Together, these results suggest that persistent stuttering in adults who stutter may be associated with reduced reliance on auditory feedback, resulting in deficits in using and integrating discrepancies between predicted and received auditory feedback to calibrate the speech production system (evidenced by a significant difference between adults who do and do not stutter, and the lack of adaptation in adults who stutter). Our results did not provide evidence for similar limitations in the auditory-motor integration of children who stutter (evidenced by a lack of significant difference between children who do and do not stutter). In fact, the suggestion of reduced reliance on auditory feedback is consistent with the results based on primarily adults who stutter, including: (a) behavioral studies that have reported reduced compensatory responses to unexpected perturbations of auditory feedback (Cai et al., 2014; Cai et al., 2012; Loucks et al., 2012; Nudelman et al., 1992); (b) neuroimaging studies that have reported reduced activation of the auditory cortex in adults who stutter (Belyk, Kraft, \& Brown, 2015; Brown, Ingham, Ingham, Laird, \& Fox, 2005; Budde, Barron, \& Fox, 2014); and (c) electrophysiological studies that have reported reduced preparation of the auditory system during speech planning as indicated by reduced modulation of auditory evoked potentials (Daliri \& Max, 2015a, 2015b; Mock, Foundas, \& Golob, 2015).

Given the significant difference between adults who stutter and those who do not stutter, and given that the stuttering children in the study are less likely to recover (as the majority of them were more than 7 years old and thus are more likely to have persistent stuttering into adulthood), one may ask why children who stutter performed similarly to children who do not stutter. One testable explanation for these results is that, over the years of stuttering, adults who stutter 
may develop a strategy in which auditory feedback is given less weight during speech production, which results in smaller adaptive responses. In other words, the reduced reliance on auditory feedback in adults who stutter may represent a secondary consequence of stuttering (or a compensatory mechanism that adults who stutter develop to cope with stuttering symptoms), and not a core deficit that leads to stuttering. Another testable explanation is related to the paradigm that we used in our study: we used a very specific and constrained paradigm with static perturbation (the perturbation was constant during a trial), which examines a very small part of the sensorimotor system (Perkell, 2012). A recent study - examining compensatory response and not adaptive response - showed that adults who stutter performed substantially different from nonstuttering adults in response to dynamic perturbations (i.e., time-varying auditory perturbations), whereas the between-group difference was less prominent in response to a static perturbation (similar to the one used in this study) (Cai et al., 2014). Thus, it can be argued that the paradigm used in the current study was not sensitive enough to detect the subtle differences between the children who stutter and children who do not stutter. Furthermore, although the children included in this study were 7 years and older and thus are those who were considered likely to have persistent stuttering, it is nevertheless possible that some of the children may eventually recover in later years. If this is the case, the difference between the control and stuttering groups would have been further attenuated. To test these explanations, future studies need to examine auditorymotor integration of children, adolescents, and adults using dynamic perturbations such as time-varying auditory perturbations.

The question remains as to what deficient neural substrates underlie these behavioral results. A growing body of evidence suggests that stuttering is associated with network-level deficits in both corticocortical loops and cortico-subcortical loops rather than an isolated deficit in one specific brain region (Cai et al., 2014; Lu et al., 2012; Neef, Anwander, \& Friederici, 2015). Studies on neurotypical adults have shown that cortico-subcortical loops are heavily involved in sensorimotor integration (Doyon, Penhune, \& Ungerleider, 2003; Doyon et al., 2009; Hardwick, Rottschy, Miall, \& Eickhoff, 2013). In addition, several studies have reported abnormalities in cortico-subcortical loops of adults who stutter (Cieslak, Ingham, Ingham, \& Grafton, 2015; Giraud et al., 2008; Lu et al., 2010). Thus, we speculate that limitations in sensorimotor adaptation of adults who stutter may result from deficits in cortico-subcortical loops. In other words, deficits in cortico-subcortical loops may affect how auditory and motor structures interconnect in adults who stutter, and limit their ability in using discrepancies between predicted and received auditory feedback to adaptively calibrate the speech motor system. However, as suggested previously, the reported limitations in integration of auditory feedback for online monitoring and corrective motor response in adults who stutter may result from deficits in cortico-cortical loops (Cai et al., 2014). It should be noted that, similar to studies on adults who stutter (Cai et al., 2014; Lu et al., 2012; Cieslak et al., 2015; Giraud et al., 2008; Lu et al., 2010), studies on children who stutter (Chang, Zhu, Choo, \& Angstadt, 2015; Chang \& Zhu, 2013; Chang et al., 2011) have also reported structural and functional abnormalities in cortico-subcortical networks involved in sensorimotor processes. However, the characteristics of these abnormalities (e.g., direction and spatial location of abnormalities relative to controls) are not the same for children and adults who stutter. For example, adults who stutter typically have over-activation in the right frontal and cerebellum areas, whereas this may not be case for children who stutter (for a review, see Neef et al., 2015). In addition, it has been suggested that the developmental trajectory of structural and/or functional characteristics of sensorimotor regions in individuals who stutter may differ from those of nonstuttering individuals (e.g., Beal et al. 2015). Therefore, it is possible that a deficient cortico-subcortical sub-network that is a part of a larger network underlying stuttering may lead to the lack of adaptation in adults who stutter, whereas the same sub-network is intact in children who stutter. Future studies will need to test these speculations by examining neural activation of people who stutter during sensorimotor adaptation and during speaking under unexpected perturbations.

In the present study, auditory feedback was experimentally manipulated but somatosensory feedback remained unaltered. We interpreted the smaller adaptive responses of adults who stutter as evidence for possible deficits in integration of auditory feedback for calibration of the speech motor system. One alternative explanation is that adults who stutter rely more on somatosensory feedback than auditory feedback, and therefore discrepancies in the auditory feedback are given lower weights in comparison to discrepancies in somatosensory feedback. In contrast to the suggestion of increased reliance on somatosensory feedback, Caruso, Gracco, and Abbs (1987) reported that the motor compensatory response to somatosensory perturbations in adults who stutter is smaller than that of nonstuttering adults, suggesting that adults who stutter have difficulties in integrating somatosensory feedback (in addition to difficulties in integrating auditory feedback). This is consistent with a large body of research that suggests that stuttering is a generalized sensorimotor disorder associated with deficits in sensory-motor (both auditory and somatosensory) integration (e.g., Daliri et al., 2013; Daliri, Prokopenko, Flanagan, \& Max, 2014; De Nil \& Abbs, 1991; Loucks \& De Nil, 2006a, 2012; Namasivayam, van Lieshout, \& De Nil, 2008). Therefore, the interpretation of increased reliance on somatosensory feedback in adults who stutter is less plausible to explain our results. However, we cannot rule out the possibility of differential weights toward somatosensory and auditory feedback in the speech motor system of adults who stutter; future studies need to systematically quantify and examine the extent of reliance on auditory feedback versus somatosensory feedback in people who stutter.

Auditory-motor learning - or more generally sensorimotor learning - is heavily dependent on sensory feedback (for a review, see Shadmehr, Smith, \& Krakauer, 2010). Thus, if stuttering is associated with reduced reliance on auditory feedback, it is reasonable to speculate that adults who stutter may have deficiencies in auditory-motor learning (as a consequence of the reduced reliance on auditory feedback). Deficiencies in auditory-motor learning in adults who stutter are supported by results of many motor learning studies of stuttering (for a review, see Namasivayam \& van Lieshout, 2011). Generally, these studies have reported that adults who stutter are slower than adults 
who do not stutter in learning speech and nonspeech movement sequences (as measured by change in reaction time and number of inaccuracies in production of a sequence). More studies are needed to further test the relationship between reliance on auditory feedback and auditory-motor learning in people who stutter.

Finally, our results showed that the adaptation magnitudes observed for children and adults who do not stutter were similar to each other; however, children who stutter showed greater adaptation magnitude than that observed in adults who stutter. Our results for children and adults who do not stutter are consistent with several previous studies of sensorimotor adaptation in the speech motor system of normally fluent speakers (MacDonald, Johnson, Forsythe, Plante, \& Munhal, 2012; Scheerer, Jacobson, \& Jones, 2016; Shiller, Gracco, \& Rvachew, 2010; Shiller \& Rochon, 2014). These studies have investigated auditory-motor adaptation (a) in response to perturbations in different parameters of speech, and (b) in normally fluent children in different age groups (compared to adults). The overall results of these studies and our own results from nonstuttering children and adults suggest that, similar to neurotypical adults, neurotypical children older than 3 years of age can integrate auditory feedback for calibration of the speech motor system; however, this may not be the case for children younger than 3 years of age. This conclusion is largely in agreement with previous suggestions that after the acquisition of speech around the age of 3 , the role of auditory feedback may gradually start to change in comparison to its role during speech acquisition (Callan, Kent, Guenther, \& Vorperian, 2000; Guenther, 2006; Kuhl, 1994; Kuhl, 2004; Tourville \& Guenther, 2011).

In summary, we used an adaptation paradigm to examine whether people who stutter have difficulties using and integrating auditory feedback for calibrating speech movements. We found that children who stutter did not differ from fluent children in integrating auditory feedback for producing an adaptive response to the auditory perturbations; however, adults who stutter were less efficient (i.e., smaller adaptation magnitude) than fluent adults in integrating auditory feedback. Our results suggest that stuttering in adults may be associated with deficits in integrating auditory feedback for updating and calibrating the speech motor system; however, the auditory-motor integration of children who stutter may be intact. Thus, this auditory-motor integration deficit in adults who stutter appears to be a compensatory effect that develops over years of stuttering.

\section{ACKNOWLEDGEMENTS}

We would like to thank Ashley Larva, Saralyn Rubsam, and other members of the Speech Neurophysiology Lab for help with speech transcriptions and disfluency analyses; as well as Kaitlyn Ayres, Kristin Hicks, and Evamarie Burnham for their help with recruitment, scheduling, and data processing. We also thank the two anonymous reviewers for their constructive critiques and helpful suggestions for improvement of the manuscript. This research was supported, in part, by grants R01DC011277 (PI: Chang) and R01DC007683 (PI: Guenther) from the National Institute on Deafness and Other Communication Disorders. The content is solely the responsibility of the authors and does not necessarily represent the official views of the National Institute on Deafness and Other Communication Disorders or the National Institutes of Health.

\section{REFERENCES}

Bauer, J.J., Seery, C.H., LaBonte, R., \& Ruhnke, L. (2007). Voice FO responses elicited by perturbations in pitch of auditory feedback in individuals that stutter and controls. Journal of the Acoustical Society of America, 121, 3201.

Bauerly, K.R., \& De Nil, L.F. (2011). Speech sequence skill learning in adults who stutter. Journal of Fluency Disorders, 36, 349-360.

Beal, D.S., Cheyne, D.O., Gracco, V.L., Quraan, M.A., Taylor, M.J., \& De Nil, L.F. (2010). Auditory evoked fields to vocalization during passive listening and active generation in adults who stutter. Neurolmage, 52, 1645-1653.

Beal, D.S., Lerch, J.P., Cameron, B., Henderson, R., Gracco, V.L., \& De Nil, L.F. (2015). The trajectory of gray matter development in Broca's area is abnormal in people who stutter. Frontiers in Human Neuroscience, 9, 89.

Belyk, M., Kraft, S.J., \& Brown, S. (2015). Stuttering as a trait or state: An ALE meta-analysis of neuroimaging studies. European Journal of Neuroscience, 41, 275-284.

Bloodstein, O., \& Bernstein-Ratner, N. (2008). A handbook on stuttering (6th edn.). Clifton Park, NY: Delmar.

Brown, S., Ingham, R., Ingham, J., Laird, A., \& Fox, P. (2005). Stuttered and fluent speech production: An ALE meta-analysis of functional neuroimaging studies. Human Brain Mapping, 25, 105-117.

Budde, K.S., Barron, D.S., \& Fox, P.T. (2014). Stuttering, induced fluency, and natural fluency: A hierarchical series of activation likelihood estimation meta-analyses. Brain and Language, 139, 99-107.

Burnett, T.A., Senner, J.E., \& Larson, C.R. (1997). Voice F-0 responses to pitch-shifted auditory feedback: A preliminary study. Journal of Voice, 11, 202-211.

Cai, S. (2015). Audapter [Computer software]. Retrieved from https:// github.com/shanqing-cai/audapter_matlab

Cai, S., Beal, D.S., Ghosh, S.S., Guenther, F.H., \& Perkell, J.S. (2014). Impaired timing adjustments in response to time-varying auditory perturbation during connected speech production in persons who stutter. Brain and Language, 129, 24-29.

Cai, S., Beal, D.S., Ghosh, S.S., Tiede, M.K., Guenther, F.H., \& Perkell, J.S. (2012). Weak responses to auditory feedback perturbation during articulation in persons who stutter: Evidence for abnormal auditorymotor transformation. PLoS ONE, 7, e41830.

Cai, S., Ghosh, S.S., Guenther, F.H., \& Perkell, J.S. (2010). Adaptive auditory feedback control of the production of formant trajectories in the Mandarin triphthong/iau/and its pattern of generalization. Journal of the Acoustical Society of America, 128, 2033-2048.

Cai, S., Ghosh, S.S., Guenther, F.H., \& Perkell, J.S. (2011). Focal manipulations of formant trajectories reveal a role of auditory feedback in the online control of both within-syllable and between-syllable speech timing. Journal of Neuroscience, 31, 16483-16490.

Cai, S., Tourville, J.A., Beal, D.S., Perkell, J.S., Guenther, F.H., \& Ghosh, S.S. (2014). Diffusion imaging of cerebral white matter in persons who stutter: Evidence for network-level anomalies. Frontiers in Human Neuroscience, 8, 54.

Callan, D.E., Kent, R.D., Guenther, F.H., \& Vorperian, H.K. (2000). An auditory-feedback-based neural network model of speech production that is robust to developmental changes in the size and shape of the articulatory system. Journal of Speech, Language, and Hearing Research, 43, 721-736

Carrow-Woolfolk, E. (1999). Test for auditory comprehension of language (3rd edn.). Circle Pines, MN: American Guidance Service.

Caruso, A.J., Gracco, V.L., \& Abbs, J.H. (1987). A speech motor control perspective on stuttering: Preliminary observations. In H.F.M. Peters \& W. 
Hulstijn (Eds.), Speech motor dynamics in stuttering (pp. 245-258). Wien, Austria: Springer Verlag.

Chang, S.E., Horwitz, B., Ostuni, J., Reynolds, R., \& Ludlow, C.L. (2011). Evidence of left inferior frontal-premotor structural and functional connectivity deficits in adults who stutter. Cerebral Cortex, 21, 2507-2518.

Chang, S.E., Kenney, M.K., Loucks, T.M.J., \& Ludlow, C.L. (2009). Brain activation abnormalities during speech and non-speech in stuttering speakers. Neurolmage, 46, 201-212.

Chang, S.E., \& Zhu, D.C. (2013). Neural network connectivity differences in children who stutter. Brain, 136, 3709-3726.

Chang, S.E., Zhu, D.C., Choo, A.L., \& Angstadt, M. (2015). White matter neuroanatomical differences in young children who stutter. Brain, 138, 694-711.

Cieslak, M., Ingham, R.J., Ingham, J.C., \& Grafton, S.T. (2015). Anomalous white matter morphology in adults who stutter. Journal of Speech, Language, and Hearing Research, 58, 268-277.

Cykowski, M.D., Fox, P.T., Ingham, R.J., Ingham, J.C., \& Robin, D.A. (2010). A study of the reproducibility and etiology of diffusion anisotropy differences in developmental stuttering: A potential role for impaired myelination. Neurolmage, 52, 1495-1504.

Daliri, A., \& Max, L. (2015a). Electrophysiological evidence for a general auditory prediction deficit in adults who stutter. Brain and Language, 150, 37-44.

Daliri, A., \& Max, L. (2015b). Modulation of auditory processing during speech movement planning is limited in adults who stutter. Brain and Language, 143, 59-68.

Daliri, A., Prokopenko, R.A., Flanagan, J.R., \& Max, L. (2014). Control and prediction components of movement planning in stuttering versus nonstuttering adults. Journal of Speech, Language, and Hearing Research, 57, 2131-2141.

Daliri, A., Prokopenko, R., \& Max, L. (2013). Afferent and efferent aspects of mandibular sensorimotor control in adults who stutter. Journal of Speech, Language, and Hearing Research, 56, 1774-1788.

De Nil, L.F., \& Abbs, J.H. (1991). Kinesthetic acuity of stutterers and nonstutterers for oral and non-oral movements. Brain, 114, 2145-2158.

Doyon, J., Bellec, P., Amsel, R., Penhune, V., Monchi, O., Carrier, J., ... \& Benali, H. (2009). Contributions of the basal ganglia and functionally related brain structures to motor learning. Behavioural Brain Research, 199, 61-75.

Doyon, J., Penhune, V., \& Ungerleider, L.G. (2003). Distinct contribution of the cortico-striatal and cortico-cerebellar systems to motor skill learning. Neuropsychologia, 41, 252-262.

Dunn, L.M., \& Dunn, D.M. (2007). PPVT-4: Peabody picture vocabulary test. Circle Pines, MN: Pearson Education.

Elman, J.L. (1981). Effects of frequency shifted feedback on the pitch of vocal productions. Journal of the Acoustical Society of America, 70, 45-50.

Feng, Y., Gracco, V.L., \& Max, L. (2011). Integration of auditory and somatosensory error signals in the neural control of speech movements. Journal of Neurophysiology, 106, 667-679.

Foundas, A.L., Mock, J.R., Corey, D.M., Golob, E.J., \& Conture, E.G. (2013). The SpeechEasy device in stuttering and nonstuttering adults: Fluency effects while speaking and reading. Brain and Language, 126, 141-150.

Fox, P., Ingham, R., Ingham, J., Hirsch, T., Downs, J., Martin, C., ... \& Lancaster, J. (1996). A PET study of the neural systems of stuttering. Nature, 382, 158-162.

Giraud, A., Neumann, K., Bachoud-Levi, A., von Gudenberg, A.W., Euler, H.A., Lanfermann, H., \& Preibisch, C. (2008). Severity of dysfluency correlates with basal ganglia activity in persistent developmental stuttering. Brain and Language, 104, 190-199.

Goldman, R., \& Fristoe, M. (2000). Goldman-Fristoe test of articulation 2 (2nd edn.). Circle Pines, MN: American Guidance Services.

Guenther, F.H. (2006). Cortical interactions underlying the production of speech sounds. Journal of Communication Disorders, 39, 350-365.

Hammill, D.D., Brown, V., Larsen, S., \& Wiederholt, J. (1994). Test of adolescent/adult language - 3. Austin, TX: Pro-Ed.
Hammill, D.D., \& Newcomer, P.L. (1988). Test of language development Intermediate. Austin, TX: Pro-Ed.

Hardwick, R.M., Rottschy, C., Miall, R.C., \& Eickhoff, S.B. (2013). A quantitative meta-analysis and review of motor learning in the human brain. Neurolmage, 67, 283-297.

Hickok, G. (2012). Computational neuroanatomy of speech production. Nature Reviews Neuroscience, 13, 135-145.

Hickok, G., Houde, J., \& Rong, F. (2011). Sensorimotor integration in speech processing: Computational basis and neural organization. Neuron, 69, 407-422.

Houde, J.F., \& Jordan, M.I. (1998). Sensorimotor adaptation in speech production. Science, 279, 1213-1216.

Houde, J.F., \& Jordan, M.I. (2002). Sensorimotor adaptation of speech I: Compensation and adaptation. Journal of Speech, Language, and Hearing Research, 45, 295-310.

Jones, J.A., \& Munhall, K.G. (2000). Perceptual calibration of FO production: Evidence from feedback perturbation. Journal of the Acoustical Society of America, 108, 1246-1251.

Jones, J.A., \& Munhall, K.G. (2002). The role of auditory feedback during phonation: Studies of Mandarin tone production. Journal of Phonetics, 30, 303-320.

Kalinowski, J., Stuart, A., Sark, S., \& Armson, J. (1996). Stuttering amelioration at various auditory feedback delays and speech rates. International Journal of Language \& Communication Disorders, 31, 259-269.

Katseff, S., Houde, J., \& Johnson, K. (2012). Partial compensation for altered auditory feedback: A tradeoff with somatosensory feedback? Language and Speech, 55, 295-308.

Kell, C.A., Neumann, K., von Kriegstein, K., Posenenske, C., von Gudenberg, A.W., Euler, H., \& Giraud, A.L. (2009). How the brain repairs stuttering. Brain, 132, 2747-2760.

Kuhl, P.K. (1994). Learning and representation in speech and language. Current Opinion in Neurobiology, 4, 812-822.

Kuhl, P.K. (2004). Early language acquisition: Cracking the speech code. Nature Reviews Neuroscience, 5, 831-843.

Lametti, D.R., Nasir, S.M., \& Ostry, D.J. (2012). Sensory preference in speech production revealed by simultaneous alteration of auditory and somatosensory feedback. Journal of Neuroscience, 32, 9351-9358.

Larson, C.R., Burnett, T.A., Bauer, J.J., Kiran, S., \& Hain, T.C. (2001). Comparison of voice $\mathrm{F}-\mathrm{O}$ responses to pitch-shift onset and offset conditions. Journal of the Acoustical Society of America, 110, 2845-2848.

Lee, B.S. (1951). Artificial stutter. Journal of Speech and Hearing Disorders, 16, 53-55.

Lincoln, M., Packman, A., \& Onslow, M. (2006). Altered auditory feedback and the treatment of stuttering: A review. Journal of Fluency Disorders, 31, 71-89.

Loucks, T., Chon, H., \& Han, W. (2012). Audiovocal integration in adults who stutter. International Journal of Language \& Communication Disorders, 47, 451-456.

Loucks, T.M.J., \& De Nil, L.F. (2006a). Oral kinesthetic deficit in adults who stutter: A target-accuracy study. Journal of Motor Behavior, 38, 238-246.

Loucks, T.M., \& De Nil, L.F. (2006b). Anomalous sensorimotor integration in adults who stutter: A tendon vibration study. Neuroscience Letters, 402, 195-200.

Loucks, T.M., \& De Nil, L.F. (2012). Oral sensorimotor integration in adults who stutter. Folia Phoniatrica et Logopaedica, 64, 116-121.

Lu, C., Chen, C., Peng, D., You, W., Zhang, X., Ding, G., ... \& Howell, P. (2012). Neural anomaly and reorganization in speakers who stutter: A shortterm intervention study. Neurology, 79, 625-632.

Lu, C., Peng, D., Chen, C., Ning, N., Ding, G., Li, K., ... \& Lin, C. (2010). Altered effective connectivity and anomalous anatomy in the basal gangliathalamocortical circuit of stuttering speakers. Cortex, 46, 49-67.

MacDonald, E.N., Goldberg, R., \& Munhall, K.G. (2010). Compensations in response to real-time formant perturbations of different magnitudes. Journal of the Acoustical Society of America, 127, 1059-1068. 
MacDonald, E.N., Johnson, E.K., Forsythe, J., Plante, P., \& Munhal, K.G. (2012). Children's development of self-regulation in speech production. Current Biology, 22, 113-117.

Max, L. (2004). Stuttering and internal models for sensorimotor control: A theoretical perspective to generate testable hypotheses. In B. Maassen, R. Kent, H.F.M. Peters, P. van Lieshout \& W. Hulstijn (Eds.), Speech motor control in normal and disordered speech (pp. 357-387). Oxford: Oxford University Press.

Max, L., Guenther, F.H., Gracco, V.L., Ghosh, S.S., \& Wallace, M.E. (2004). Unstable or insufficiently activated internal models and feedback-biased motor control as sources of dysfluency: A theoretical model of stuttering. Contemporary Issues in Communication Science and Disorders, 31, 105-122.

Max, L., \& Maffett, D.G. (2015). Feedback delays eliminate auditory-motor learning in speech production. Neuroscience Letters, 591, 25-29.

Max, L., \& Onghena, P. (1999). Some issues in the statistical analysis of completely randomized and repeated measures designs for speech, language, and hearing research. Journal of Speech, Language, and Hearing Research, 42, 261-270.

Mayer, M. (1969). Frog, where are you?. New York: Dial Press.

Mock, J.R., Foundas, A.L., \& Golob, E.J. (2015). Speech preparation in adults with persistent developmental stuttering. Brain and Language, 149, 97-105.

Namasivayam, A.K., \& van Lieshout, P. (2011). Speech motor skill and stuttering. Journal of Motor Behavior, 43, 477-489.

Namasivayam, A.K., van Lieshout, P., \& De Nil, L. (2008). Bite-block perturbation in people who stutter: Immediate compensatory and delayed adaptive processes. Journal of Communication Disorders, 41, 372-394.

Neef, N.E., Anwander, A., \& Friederici, A.D. (2015). The neurobiological grounding of persistent stuttering: From structure to function. Current Neurology and Neuroscience Reports, 15, 63.

Newcomer, P.L., \& Hammill, D.D. (1977). Test of language development. Austin, TX: Empiric Press.

Nudelman, H.B., Herbrich, K.E., Hess, K.R., Hoyt, B.D., \& Rosenfield, D.B. (1992). A model of the phonatory response time of stutterers and fluent speakers to frequency-modulated tones. Journal of the Acoustical Society of America, 92, 1882-1888.

Olander, L., Smith, A., \& Zelaznik, H.N. (2010). Evidence that a motor timing deficit is a factor in the development of stuttering. Journal of Speech, Language, and Hearing Research, 53, 876-886.

Oldfield, R.C. (1971). The assessment and analysis of handedness: The Edinburgh inventory. Neuropsychologia, 9, 97-113.

Perkell, J.S. (2012). Movement goals and feedback and feedforward control mechanisms in speech production. Journal of Neurolinguistics, 25, 382-407.

Purcell, D.W., \& Munhall, K.G. (2006a). Compensation following real-time manipulation of formants in isolated vowels. Journal of the Acoustical Society of America, 119, 2288-2297.

Purcell, D.W., \& Munhall, K.G. (2006b). Adaptive control of vowel formant frequency: Evidence from real-time formant manipulation. Journal of the Acoustical Society of America, 120, 966-977.

Quinn, G.P., \& Keough, M.J. (2002). Experimental design and data analysis for biologists. Cambridge: Cambridge University Press.

Riley, G.D. (2008). SSI-4, Stuttering severity instrument for children and adults (4th edn.). Austin, TX: Pro Ed.

Rochet-Capellan, A., Richer, L., \& Ostry, D.J. (2012). Nonhomogeneous transfer reveals specificity in speech motor learning. Journal of Neurophysiology, 107, 1711-1717.

Ruxton, G.D., \& Beauchamp, G. (2008). Time for some a priori thinking about post hoc testing. Behavioral Ecology, 19, 690-693.

Sasisekaran, J., Smith, A., Sadagopan, N., \& Weber-Fox, C. (2010). Nonword repetition in children and adults: Effects on movement coordination. Developmental Science, 13, 521-532.

Scheerer, N.E., Jacobson, D.S., \& Jones, J.A. (2016). Sensorimotor learning in children and adults: Exposure to frequency-altered auditory feedback during speech production. Neuroscience, 314, 106-115.
Shadmehr, R., Smith, M.A., \& Krakauer, J.W. (2010). Error correction, sensory prediction, and adaptation in motor control. Annual Review of Neuroscience, 33, 89-108.

Shiller, D.M., Gracco, V.L., \& Rvachew, S. (2010). Auditory-motor learning during speech production in 9-11-year-old children. PLOS ONE, 5, e12975.

Shiller, D.M., \& Rochon, M. (2014). Auditory-perceptual learning improves speech motor adaptation in children. Journal of Experimental Psychology: Human Perception and Performance, 40, 1308-1315.

Smits-Bandstra, S., \& De Nil, L.F. (2007). Sequence skill learning in persons who stutter: Implications for cortico-striato-thalamo-cortical dysfunction. Journal of Fluency Disorders, 32, 251-278.

Smits-Bandstra, S., \& De Nil, L.F. (2009). Speech skill learning of persons who stutter and fluent speakers under single and dual task conditions. Clinical Linguistics \& Phonetics, 23, 38-57.

Smits-Bandstra, S., De Nil, L.F., \& Rochon, E. (2006). The transition to increased automaticity during finger sequence learning in adult males who stutter. Journal of Fluency Disorders, 31, 22-42.

Smits-Bandstra, S., De Nil, L.F., \& Saint-Cyr, J.A. (2006). Speech and nonspeech sequence skill learning in adults who stutter. Journal of Fluency Disorders, 31, 116-136.

Sommer, M., Koch, M., Paulus, W., Weiller, C., \& Buchel, C. (2002). Disconnection of speech-relevant brain areas in persistent developmental stuttering. Lancet, 360, 380-383.

Tourville, J.A., \& Guenther, F.H. (2011). The DIVA model: A neural theory of speech acquisition and production. Language and Cognitive Processes, 26, 952-981.

Tourville, J.A., Reilly, K.J., \& Guenther, F.H. (2008). Neural mechanisms underlying auditory feedback control of speech. Neurolmage, 39, 1429-1443.

Vaughn, C., \& Nasir, S.M. (2015). Precise feedback control underlies sensorimotor learning in speech. Journal of Neurophysiology, 113, 950-955.

Villacorta, V.M., Perkell, J.S., \& Guenther, F.H. (2007). Sensorimotor adaptation to feedback perturbations of vowel acoustics and its relation to perception. Journal of the Acoustical Society of America, 122, 2306-2319.

Watkins, K.E., Smith, S.M., Davis, S., \& Howell, P. (2008). Structural and functional abnormalities of the motor system in developmental stuttering. Brain, 131, 50-59.

Wechsler, D. (1999). Wechsler abbreviated scale of intelligence. New York: The Psychological Corporation.

Williams, K.T. (2007). Expressive vocabulary test (2nd edn.). Circle Pines, MN: American Guidance Services.

Wingate, M. (1969). Sound and pattern in 'artificial' fluency. Journal of Speech, Language, and Hearing Research, 12, 677-686.

Wymbs, N.F., Ingham, R.J., Ingham, J.C., Paolini, K.E., \& Grafton, S.T. (2013). Individual differences in neural regions functionally related to real and imagined stuttering. Brain and Language, 124, 153-164.

Yairi, E., \& Ambrose, N.G. (1999). Early childhood stuttering I: Persistency and recovery rates. Journal of Speech, Language, and Hearing Research, 42, 1097-1112.

Yairi, E., \& Ambrose, N. (2013). Epidemiology of stuttering: 21st century advances. Journal of Fluency Disorders, 38, 66-87.

Yates, A.J. (1963). Delayed auditory feedback. Psychological Bulletin, 60, 213-232.

How to cite this article: Daliri A, Wieland EA, Cai S, Guenther $\mathrm{FH}$, and Chang S-E. Auditory-motor adaptation is reduced in adults who stutter but not in children who stutter. Dev Sci. 2018;21:e12521. https://doi.org/10.1111/desc.12521 